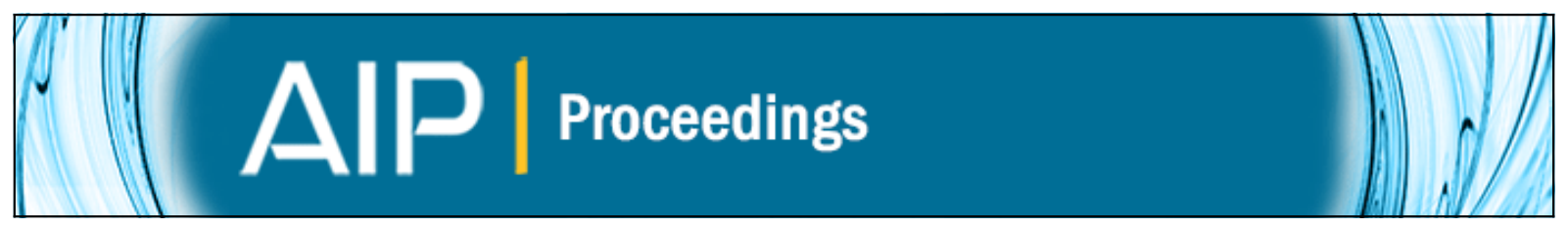

Molecular dynamics simulation of deposition and growth of $\mathrm{Cu}$ thin film on Si substrate Jun Zhang, Chong Liu, Yonghua Shu, and Jing Fan

Citation: AIP Conference Proceedings 1501, 919 (2012); doi: 10.1063/1.4769640

View online: http://dx.doi.org/10.1063/1.4769640

View Table of Contents: http://scitation.aip.org/content/aip/proceeding/aipcp/1501?ver=pdfcov

Published by the AIP Publishing

Articles you may be interested in

Large-scale molecular dynamics simulations of glancing angle deposition

J. Appl. Phys. 114, 083517 (2013); 10.1063/1.4819446

Molecular dynamics simulation of the thin film deposition of $\mathrm{Co} / \mathrm{Cu}(111)$ with $\mathrm{Pb}$ surfactant

J. Appl. Phys. 106, 044304 (2009); 10.1063/1.3194309

Copper enhanced (111) texture in silver thin films on amorphous Si O 2

J. Appl. Phys. 102, 083548 (2007); 10.1063/1.2800998

Atomistic mechanisms of strain relaxation due to ductile void growth in ultrathin films of face-centered-cubic metals

J. Appl. Phys. 97, 113527 (2005); 10.1063/1.1926393

Stress evolution during and after sputter deposition of Cu thin films onto Si (100) substrates under various sputtering pressures

J. Appl. Phys. 97, 054908 (2005); 10.1063/1.1858062 


\title{
Molecular Dynamics Simulation of Deposition and Growth of Cu Thin Film on Si Substrate
}

\author{
Jun Zhang, Chong Liu, Yonghua Shu and Jing Fan \\ State Key Laboratory of High-temperature Gas Dynamics, Institute of Mechanics, \\ Chinese Academy of Sciences, Beijing 100190, China
}

\begin{abstract}
Growth and properties of $\mathrm{Cu}$ thin film deposited on Si substrate is studied using molecular dynamics method. Tersoff potential parameters for the interaction between $\mathrm{Cu}$ and $\mathrm{Si}$ are fitted to reproduce the lattice structure of copper silicide. We focus on the growth mode, crystalline structure and orientation, and surface morphology of $\mathrm{Cu}$ thin film. The effect of substrate temperature on the crystalline orientation and surface roughness is studied.
\end{abstract}

Keywords: Thin film growth, crystalline structure and orientation, surface roughness, molecular dynamics PACS: 68.55.-a, 68.35.Ct

\section{INTRODUCTION}

Physical vapor deposition (PVD) technique has been widely used for thin film technology, such as coating of high temperature superconducting film, metallic coating of fibers, etc. The general PVD processes involve three steps: excitation of source materials, transport of vapor atoms or molecules to substrate and film deposition on substrate. In order to optimize process conditions, knowledge of the underlying mechanism of vapor transport and film deposition is needed. The vapor transport process is usually performed under highly rarefied conditions, and hence the direct simulation Monte Carlo (DSMC) method is very efficient to simulate this process. It is recognized that the film deposition and growth process is directly related to the properties of thin film, so it is preferred to employ molecular dynamics (MD) simulation to examine the film growth process and to analyze the morphology of the deposited thin film in detail.

Recently, $\mathrm{Cu}$ has been gradually used as interconnection material in integrated circuits to replace $\mathrm{Al}$ due to its low resistivity and high electron migration resistance. This replacement results in the research interests of epitaxial growth of $\mathrm{Cu}$ thin film on Si substrate. Many efforts have been devoted to depositing $\mathrm{Cu}$ atoms on $\mathrm{Si}$ substrate using various methods such as molecular beam epitaxy [1], electron beam evaporation [2,3] and magnetron sputtering [4]. In addition, the study of metal/semiconductor interfaces has been of crucial importance in micro-electronic devices due to their key role in the establishment of an ohmic contact, which is usually required for semiconductor electronic circuitry. Therefore, the chemical and physical properties of metal/semiconductor couples have been intensively studied $[5,6]$.

Until now, most of studies of $\mathrm{Cu}$ thin films on Si substrate were performed by experimental methods. Due to the limitation of experiment conditions, these studies were limited to some specific cases, not systematical study. In this paper, we simulate the deposition and growth of $\mathrm{Cu}$ thin film using MD method. Our simulation corresponds to the experiment of electron beam physical vapor deposition (EBPVD). The growth mode, crystalline structure and orientation, and surface roughness of $\mathrm{Cu}$ thin film deposited and grown on $\mathrm{Si}$ substrate under different substrate temperature conditions are investigated in detail. Our objective is to provide useful information for the production of high-quality $\mathrm{Cu}$ thin film on $\mathrm{Si}$ substrate.

\section{MOLECULAR DYNAMICS METHOD AND SIMULATION MODEL}

In MD simulations, potential function and corresponding parameters describing interactions between atoms are very crucial for accurately predict properties of materials. So far, many-body empirical potentials have been constructed for metallic and covalent systems. The embedded-atom method (EAM) potential proposed by Daw and Baskes [7] gives a realistic description behavior and properties of metallic bonding. For covalent bonding, the widely used potential for semiconductors was developed by taking into account the effects of coordination-number changes on the local short-range environment of the covalent bonds. In this work, the EAM potential and

(C) 2012 American Institute of Physics 978-0-7354-1115-9/\$30.00 
corresponding parameters developed by Adams et al. [8] are utilized to describe the atomic interaction of $\mathrm{Cu}$ atoms, and Tersoff potential $[9,10]$ is used for the interaction of $\mathrm{Si}$ atoms. For the interaction between $\mathrm{Si}$ and $\mathrm{Cu}$ atoms, the effect of variable charge transfer also plays an important role. Recently, the extended Tersoff potential [11]and Charge optimized many-body (COMB) potential [12] have been developed by adding terms corresponding to the effects of charge transfer. However, the applications of these potentials to thin film deposition may be limited due to the fact that they require significantly larger computational efforts than traditional potentials. For example, the computation cost of COMB potential is as much as 10 times that of the EAM and traditional Tersoff potentials for the same simulation conditions. Therefore, we employ the traditional Tersoff potential to describe the interaction between $\mathrm{Si}$ and $\mathrm{Cu}$ atoms. Charge transfer between $\mathrm{Cu}$ and $\mathrm{Si}$ is effectively taken into account through the parameter $\chi_{C u-S i}$, which is fitted to reproduce the lattice structure of copper silicide. The Tersoff potential parameters we used are given in Table 1 .

TABLE 1. Parameter values for Tersoff potential.

\begin{tabular}{ccc}
\hline & $\mathbf{S i}$ & $\mathbf{C u}$ \\
\hline$A(\mathrm{eV})$ & 1830.8 & 803.12 \\
$B(\mathrm{eV})$ & 471.18 & 43.25 \\
$\lambda\left(\AA^{-1}\right)$ & 2.4799 & 2.83 \\
$\mu\left(\AA^{-1}\right)$ & 1.7322 & 1.412 \\
$\beta$ & $1.1000 \mathrm{E}-6$ & 0 \\
$n$ & 0.78734 & 1 \\
$c$ & $1.0039 \mathrm{E} 5$ & 0 \\
$d$ & 16.217 & 1 \\
$h$ & -0.59825 & 0 \\
$R(\AA)$ & 2.85 & 3.075 \\
$D(\AA)$ & 0.15 & 0.255 \\
& \multicolumn{2}{c}{$\chi_{C u-S i}=1.02239$} \\
\hline
\end{tabular}

Figure 1 shows the model system which consists of two parts, namely the Si substrate and the deposited $\mathrm{Cu}$ atoms. The Si substrate has the dimensions of $20 a \times 20 a \times 6 a(a=5.43 \AA$, lattice constant of $\mathrm{Si})$ and consists of 19,200 atoms with diamond structure. The (001) plane of substrate is perpendicular to the z-axis. There are three types of $\mathrm{Si}$ atoms in substrate, namely fixed atoms, thermostat atoms, and Newtonian atoms. The fixed atoms comprise the bottom 4 layers of atoms, which are fixed on their perfect lattice sites to prevent the moving of the substrate by the incident atoms during deposition. The middle 12 layers of atoms are defined as the thermostat atoms, which are used to eliminate the increasing of substrate temperature due to the hit of incident atoms during deposition process. The Newtonian atoms comprise the top 8 layers of substrate atoms. The motions of thermostat atoms and Newtonian atoms are determined by the Newton's equations of motion, besides the atom velocities of thermostat layers are rescaled at every 10 time steps according to the prescribed substrate temperature. In our simulations, the equations of motion are solved using Verlet time-integration algorithm with a constant time step of $\Delta t=1 \mathrm{fs}$.

Deposition is performed by depositing one $\mathrm{Cu}$ atom every 400 calculating time steps, which means that the deposition rate is $2.5 \mathrm{atoms} / \mathrm{ps}$. The coordinates of deposited atoms are randomly generated within the insertion volume, which has the dimensions of $20 a \times 20 a \times 6 a$ and is $10 a$ height above the substrate surface. The initial velocities of deposited atoms are composed of macroscopic velocity and thermal velocity. In our simulations, the macroscopic velocities are chosen as $550 \mathrm{~m} / \mathrm{s}$ vertically toward the substrate surface, while the thermal velocities are randomly selected from a Maxwellian distribution at the temperature of $220 \mathrm{~K}$. These values are selected according to the general results in EBPVD experiments [13]. Periodic boundary conditions are assumed in the $\mathrm{x}$ and $\mathrm{y}$ directions. Each simulation case proceeds until 22,000 $\mathrm{Cu}$ atoms are deposited on Si substrate and then a relaxation process of $1200 \mathrm{ps}$ is conducted to enable the deposited thin film to be equilibrated. All of our MD simulations are performed using classic MD package-LAMMPS [14]. 


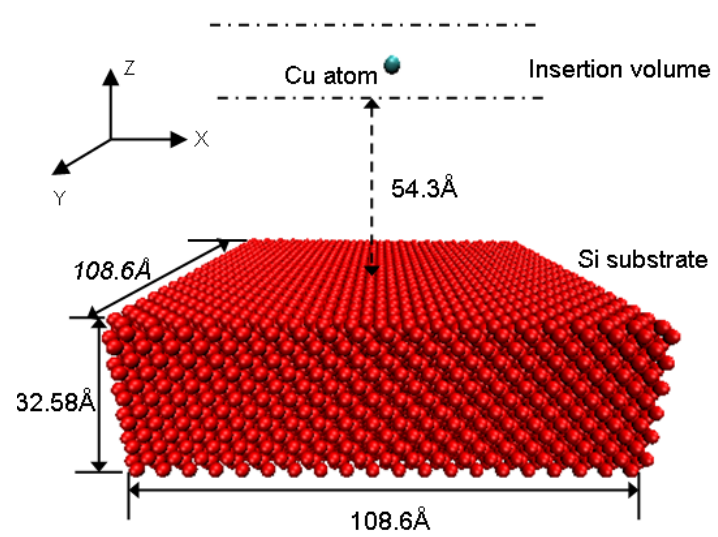

FIGURE 1. Simulation model for deposition of $\mathrm{Cu}$ atom on Si substrate.

\section{RESULTS AND DISCUSSION}

In this section we present our simulation results. The growth mode, crystalline structure and orientation, and surface morphology of the deposited $\mathrm{Cu}$ thin film are investigated in detail.

\section{Growth Mode}

Figure 2 shows the snapshots when 1250 and 10,000 $\mathrm{Cu}$ atoms (denoted in cyan) are deposited on the substrate (denoted in red) with the temperature of $300 \mathrm{~K}$, respectively. It is shown that the 1,250 deposited Cu atoms (Fig. 2a) form small three-dimensional clusters with some substrate atoms still exposed, and the surface morphology of 10,000 atoms deposited film (Fig. 2b) is with roughness in some extent. To quantify the growth process of $\mathrm{Cu}$ film in detail, the density profile of $\mathrm{Cu}$ atoms along $\mathrm{z}$ direction is shown in Fig. 3. For the sake of clarity, the original surface of $\mathrm{Si}$ substrate is shown in Fig. 3 as black dashed line. At the early stage (250 $\mathrm{Cu}$ atoms deposited), the $\mathrm{Cu}$ atoms are randomly adsorbed on the surface of $\mathrm{Si}$ substrate. After $1250 \mathrm{Cu}$ atoms are deposited, small islands are formed (Fig. 2a). From then on, film growth continues through the nucleation and coalescence of these islands, which can be explained by the Volmer-Weber growth mode [15], i.e., island growth mode. The substrate surface is completely covered by $\mathrm{Cu}$ atoms when $5000 \mathrm{Cu}$ atoms are deposited. Simultaneously, due to the diffusion mechanism, some $\mathrm{Cu}$ atoms have gotten across the original surface of Substrate as shown in Fig. 3. It can also be seen from Fig. $2 \mathrm{~b}$ that interspecies mixing occurs at the interface between $\mathrm{Cu}$ film and $\mathrm{Si}$ substrate. This mixing phenomenon between $\mathrm{Cu}$ film and $\mathrm{Si}$ substrate has been observed in experiments by Echigoya et al. [5] and Chen et al. [16] They further found that copper silicide was formed at the interface due to interfacial reaction.

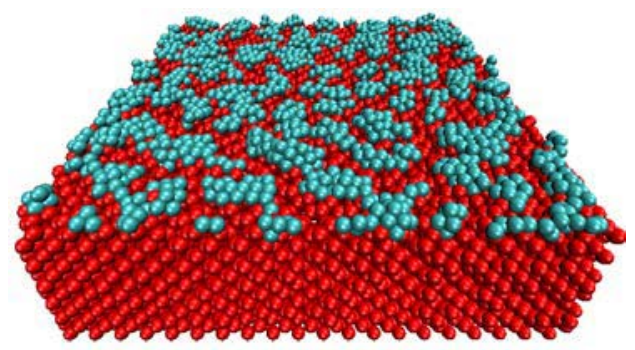

(a)

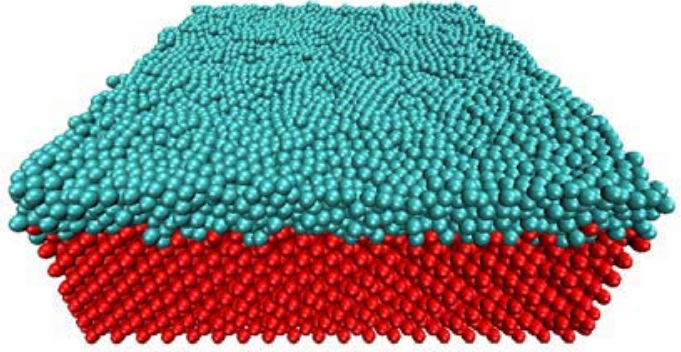

(b)

FIGURE 2. Morphology of deposited $\mathrm{Cu}$ film in the growth process: (a) $1250 \mathrm{Cu}$ atoms deposited, and (b) 10,000 $\mathrm{Cu}$ atoms deposited. 


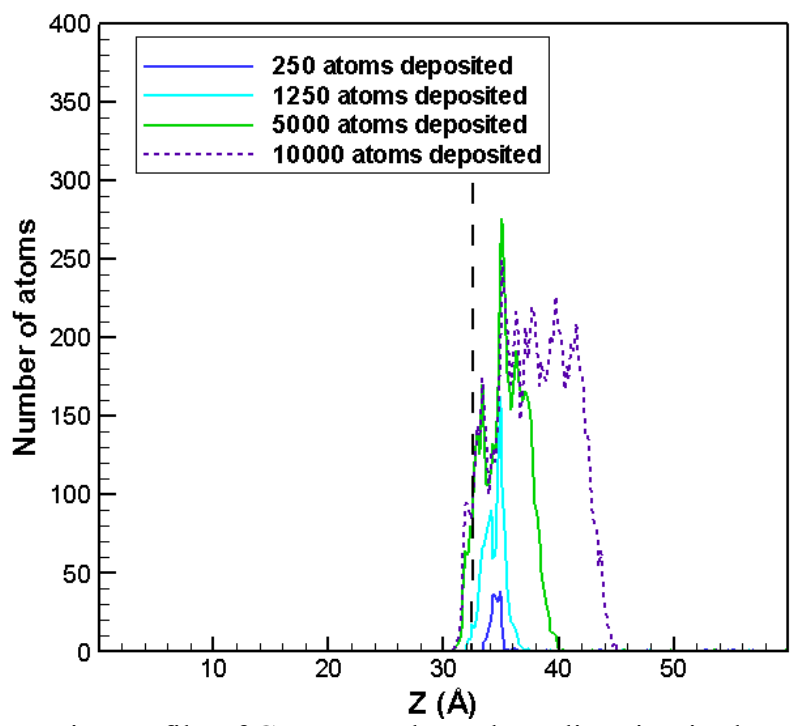

FIGURE 3. Density profile of $\mathrm{Cu}$ atoms along the $\mathrm{z}$ direction in the growth process.

\section{Crystalline Structure and Orientation}

Crystalline structure and orientation are the most important properties for deposited thin film. We employ the common neighbor analysis (CNA) method to analyze the local crystal structure of the deposited Cu film. The CNA method, firstly proposed by Honeycutt and Andersen [17], is a decomposition of the pair distribution function (PDF) according to the environment of the pairs and can provide direct interpretation of various features of PDF in terms of atomic structure. Faken and Jonsson [18] generalized such a method and systematically analyzed the local atomic structure of a molten $\mathrm{Cu}$ slab in combination with three-dimensional computer graphics. Using CNA analysis, we identified the $\mathrm{Cu}$ atoms in three different kinds of environment: face-centered cubic (fcc) structure, hexagonal closepacked (hcp) structure and amorphous structure. The atomic configurations at the particular cross section $(\mathrm{z}=50 \AA)$ are shown in Fig. 4 for substrate temperature $\mathrm{T}=300 \mathrm{~K}$ and $\mathrm{T}=900 \mathrm{~K}$. Atoms are colored according to their CNA values: dark blue denotes fcc structure, cyan denotes hep structure and read denotes amorphous structure. A very interesting phenomenon is noted that for $\mathrm{T}=300 \mathrm{~K}$ the fcc structure is $(001)$ oriented with a rotation by $45^{\circ}$ along the $<001>$ axis of substrate, while for $\mathrm{T}=900 \mathrm{~K}$ the fcc structure is (111) oriented. It is known that for Cu crystal with fcc structure, the (001) planes have the highest relative surface energy, while the closed-packed (111) planes have the lowest relative surface energy. Therefore, deposited $\mathrm{Cu}$ film naturally prefer to grow with a (111) orientation to reduce surface free energy. However, in our MD simulations, the deposition rate is one atom per $0.4 \mathrm{ps}$, which means that one monolayer of $\mathrm{Cu}$ film is covered on substrate per $7.2 \times 10^{-10} \mathrm{~s}$. If the deposited atoms are desired to form (111) oriented structure, there is at least one effective jump for each atom within $7.2 \times 10^{-10} \mathrm{~s}$. The mean time of one effective jump could be evaluated as follows:

$$
\tau=\frac{1}{v_{0}} \exp (\varepsilon / k T),
$$

where $v_{0}$ is vibration frequency, $\varepsilon$ is activation energy and $k$ is Boltzmann constant. For metals with fcc structure typical values give as $v_{0} \approx 10^{12} \mathrm{~s}^{-1}$ and $\varepsilon \approx 0.3 \mathrm{eV}$. According to Eq. (1), the mean time of one effective jump for $\mathrm{T}=300 \mathrm{~K}$ is about $1.1 \times 10^{-7} \mathrm{~s}$, which is several orders larger than the time within which one monolayer is formed. This means that most of $\mathrm{Cu}$ atoms could not jump to their preferred locations and only vibrate in the neighborhood of their initial locations. For this reason, it is possible that (001) oriented $\mathrm{Cu}$ film grows on (001) Si substrate for $\mathrm{T}=300 \mathrm{~K}$. The rotation by $45^{\circ}$ along $<001>$ axis is governed by the geometrical lattice match rule. $\mathrm{Cu}$ has an fcc structure with a lattice constant of $3.615 \mathrm{~A}$, while $\mathrm{Si}$ has a diamond structure with a lattice constant of 5.43A. If the (001) oriented $\mathrm{Cu}$ film directly grows on the (001) plane of Si substrate, the lattice mismatch is $33 \%$, which is much larger than the critical lattice parameter mismatch of $15 \%$ for epitaxial growth. However, if the $(001)$ oriented $\mathrm{Cu}$ film rotates $45^{\circ}$ along $<001>$ axis, the lattice mismatch will decrease to $5.7 \%$. In this way, it is applicable for (001) oriented $\mathrm{Cu}$ film epitaxially grows on the (001) plane of Si substrate. When the substrate temperature increases to 
$900 \mathrm{~K}$, the mean time of one effective jump is about $4.8 \times 10^{-11} s$, which is one order smaller than the time within which one monolayer is formed. Consequently, there are enough effective jumps for deposited atoms to move to their preferred locations and form (111) oriented structure.

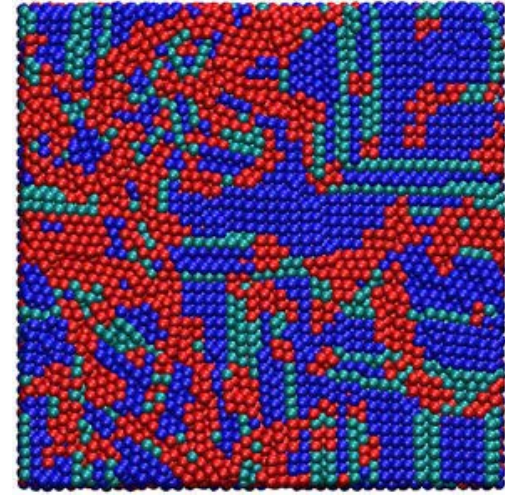

(a)

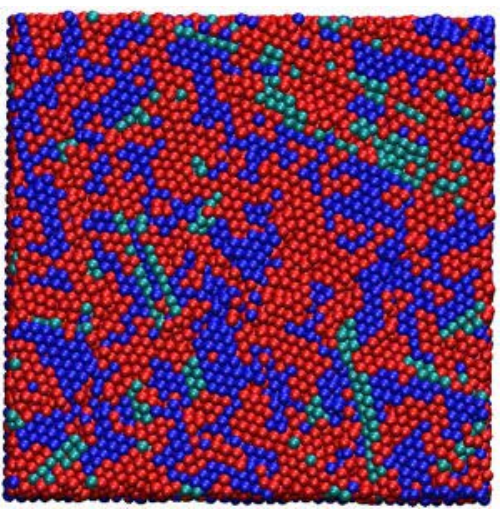

(b)

FIGURE 4. Atomic configurations at the cross-section of $z=50 \AA$ : (a) substrate temperature is $300 \mathrm{~K}$; (b) substrate temperature is $900 \mathrm{~K}$.

\section{Surface Roughness}

Besides crystalline structure and orientation, the quality and property of the deposited films is also closely related to the surface morphology. For example, Timoshevskii et al. [19] demonstrated by an ab initio study that atomicscale surface roughness (1-3 atoms) on a perfect copper surface could lead to a substantial $(30 \%-40 \%)$ reduction in the electrical conductivity of thin $\mathrm{Cu}$ film. The physical origin of roughness-induced conductivity reduction may relate to the destruction of isotropic Fermi surface sheets. Figure 5 shows the surface morphology of deposited thin film at final state under different substrate temperature. It is obvious that the surface at $900 \mathrm{~K}$ is much smoother than that at $300 \mathrm{~K}$. The surface roughness is quantitatively evaluated using the definition of root-mean-square roughness:

$$
R_{s}=\sqrt{\frac{\sum_{i=1}^{n}\left(Z_{i}-\bar{Z}\right)^{2}}{n}},
$$

where $Z_{i}$ represents the height of the exposed atoms on the film surface, $\bar{Z}$ is the mean height of all the exposed atoms, and $n$ is the total number of the exposed atoms. Figure 6 shows the surface roughness under different substrate temperature according to Eq. (2). It is shown that the surface roughness decreases with the increasing of temperature. This phenomenon is directly related to surface diffusion. Although surface diffusion coefficient is difficult to well-quantified, an empirical relationship for fcc metal surfaces is popularly used:

$$
D_{s}=D_{0} \exp \left(-E_{d} / k T\right),
$$

where $D_{0}$ is the pre-exponential factor, and $E_{d}$ is the activation energy. If $D_{0}$ and $E_{d}$ keep invariant, $D_{s}$ will increase as the increasing of $T$. In addition, we have showed in the previous subsection that the surface structure changes from (001) oriented to (111) oriented with the increasing of temperature. Usually, the activation energy for (111) plane is smaller than that for (001) plane. For these two reasons, surface diffusion coefficient increases as the increasing of temperature, and thus the surface of thin film becomes smoother. 


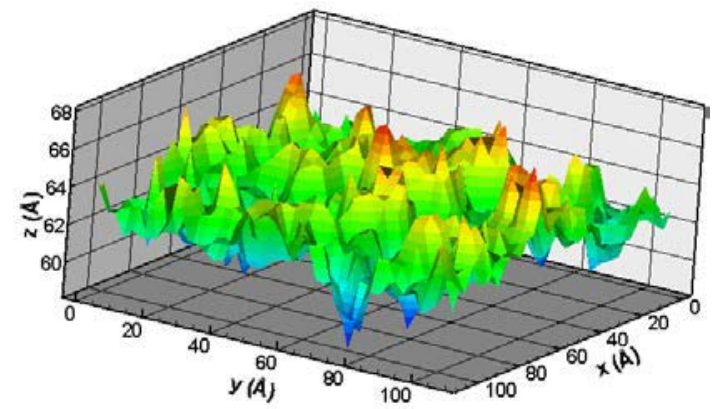

(a)

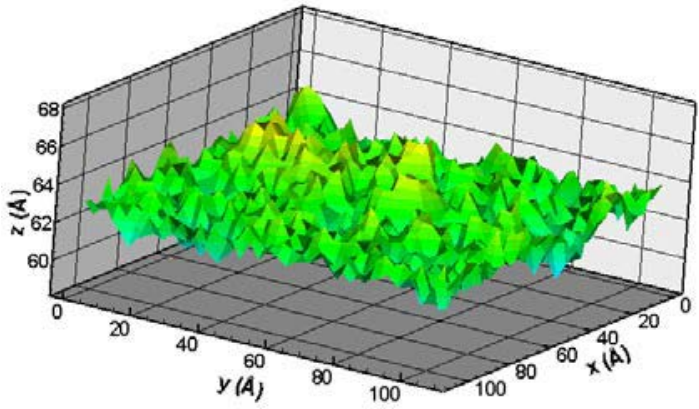

(b)

FIGURE 5. Surface morphology of $\mathrm{Cu}$ thin film at the final state: (a) substrate temperature is $300 \mathrm{~K}$; (b) substrate temperature is $900 \mathrm{~K}$.

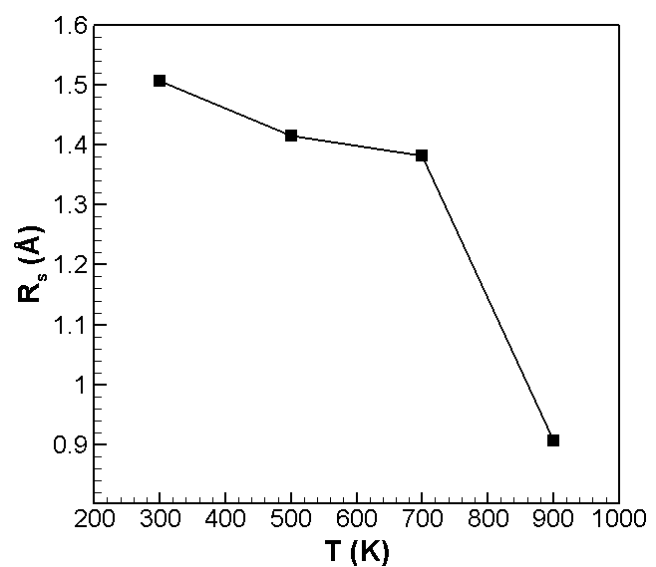

FIGURE 6. Surface roughness versus substrate temperature.

\section{CONCLUSIONS}

The present work studied the deposition and growth of $\mathrm{Cu}$ thin film on $\mathrm{Si}(001)$ substrate using molecular dynamics method. Growth mode, crystalline structure and orientation, and surface morphology of $\mathrm{Cu}$ thin film are investigated in detail. Our simulation results show that the growth of $\mathrm{Cu}$ thin film on Si substrate is threedimensional island growth mode. Interspecies mixing occurs at the interface between $\mathrm{Cu}$ film and $\mathrm{Si}$ substrate. When the substrate temperature is $300 \mathrm{~K}$, the formed fcc structure of $\mathrm{Cu}$ thin film is $(001)$ oriented with a rotation by $45^{\circ}$ along $<001>$ axis of substrate. When the substrate temperature is $900 \mathrm{~K}$, the formed fcc structure of $\mathrm{Cu}$ thin film becomes to be (111) oriented. Surface roughness decreases as the increasing of substrate temperature. Knowledge of how the substrate temperature influences film property allows for better control over the production of $\mathrm{Cu}$ thin film on Si substrate.

\section{ACKNOWLEDGMENTS}

This work was supported by the National Natural Science Foundation of China (Grant Nos. 11002147 and 10921062).

\section{REFERENCES}

1. B. G. Demczyk, R. Naik, G. Auner, C. Kota, and U. Rao, J. Appl. Phys. 75, 1956-1961 (1994).

2. C. A. Chang, J. Appl. Phys. 67, 566-569 (1990).

3. C. A. Chang, J. C. Liu, and J. Angilello, Appl. Phys. Lett. 57, 2239-2240 (1990).

4. H. Jiang, T. J. Klemmer, J. A. Barnard, and E. A. Payzant, J. Vac. Sci. Technol. A 16, 3376-3383 (1998).

5. J. Echigoya, H. Enoki, T. Satoh, T. Waki, T. Ohmi, M. Otsuki, and T. Shibata, Appl. Surf. Sci. 56-8, 463468 (1992).

6. C. A. F. Vaz, S. J. Steinmuller, C. Moutafis, J. A. C. Bland, and A. Y. Babkevich, Surf. Sci. 601, 1377-1383 (2007). 
7. M. S. Daw, and M. I. Baskes, Phys. Rev. B 29, 6443-6453 (1984).

8. J. B. Adams, S. M. Foiles, and W. G. Wolfer, J. Mater. Res. 4, 102-112 (1989).

9. J. Tersoff, Phys. Rev. B 37, 6991-7000 (1988).

10. J. Tersoff, Phys. Rev. B 39, 5566-5568 (1989).

11. A. Yasukawa, JSME Int. J. Ser. A-Mech. Mater. Eng. 39, 313-320 (1996).

12. T.-R. Shan, B. D. Devine, S. R. Phillpot, and S. B. Sinnott, Phys. Rev. B 83, 115327 (2011).

13. J. Fan, I. D. Boyd, and C. Shelton, J. Vac. Sci. Technol. A 18, 2937-2945 (2000).

14. S. Plimpton, J. Comput. Phys. 117, 1-19 (1995).

15. M. Volmer, and A. Weber, Z. Phys. Chem.-Stochiometrie Und Verwandtschaftslehre 119, 277-301 (1926).

16. L. J. Chen, C. S. Liu, and J. B. Lai, Mater. Sci. Semicond. Process 7, 143-156 (2004).

17. J. D. Honeycutt, and H. C. Andersen, J. Phys. Chem. 91, 4950-4963 (1987).

18. D. Faken, and H. Jonsson, Comput. Mater. Sci. 2, 279-286 (1994).

19. V. Timoshevskii, Y. Ke, H. Guo and D. Gall, J. Appl. Phys. 103, 113705 (2008). 